\title{
Near-Field Emission of Lead-Sulfide-Selenide Homojunction Lasers
}

\author{
H. J. KIMBLE
}

\begin{abstract}
Measurements of the near-field intensity distributions of three lead-sulfide-selenide diode lasers operating near $4.8 \mu \mathrm{m}$ have been made as a function of injection current. Localized emission in the near field exhibits peaked structure of full width from 5 to $10 \mu \mathrm{m}$ for operation above threshold. From the dependence of the emission profiles on injection current estimates of $25 \mathrm{~cm}^{-1}$ and $0.09 \mathrm{~cm} / \mathrm{A}$ are made for the distributed loss and gain coefficients for one of the lasers. Optical confinement perpendicular to the p-n junction can be explained in terms of the homojunction properties.
\end{abstract}

I JECTION lasers formed from IV-VI compounds are utilized in an increasing number of experiments in high resolution spectroscopy in the range of wavelengths from 4 to $11 \mu \mathrm{m}$. However, in contrast to the situation that exists for the gallium-arsenide laser, relatively little information is available for the IV-VI compounds about such basic properties of the laser as the relationship of gain to injection current, the nature and magnitude of the distributed losses, or the guiding mechanism within the laser cavity. With the exception of the work by Ralston et al. [1], no measurements of the nearfield intensity distributions for lead-salt lasers appear in the literature. This report describes our observations of the nearfield emissions of three lead-sulfide-selenide homojunction lasers.

The fabrication of the $\mathrm{PbS}_{1-x} \mathrm{Se}_{x}$ lasers is as described by Lo and Swets [2], with the modifications that for our lasers $x=$ 0.18 and there is no gradient in concentration in the p-type material other than that produced by the diffusion process used to form the p-n junction, which has a depth of 10-15 $\mu \mathrm{m}$ from the n-type side. Three devices I, II, and III were cleaved from the same source material and have lengths of 350,355 , and $380 \mu \mathrm{m}$, widths along the p-n junction of 170 , 178 , and $125 \mu \mathrm{m}$, and thicknesses perpendicular to the junction plane of 300,330 , and $200 \mu \mathrm{m}$, respectively. Cleaved facets form the Fabry-Perot cavity mirrors, and the emission frequency for all devices is about $63 \mathrm{Thz}\left(2100 \mathrm{~cm}^{-1}\right)$.

The three lasers are mounted in turn on a copper cold-finger that is held near $12 \mathrm{~K}$ by a dewar filled with liquid helium. The tailpiece of the dewar has been mechanically stabilized, and interferometric tests indicate that the diode position on the cold-finger is stable to $\pm 0.05 \mu \mathrm{m}$ over an interval of a few tenths of a second and to $\pm 0.15 \mu \mathrm{m}$ over several minutes. A $\mathrm{CaF}_{2}$ window mounted perpendicular to the laser axis allows collection of the laser radiation lying in a cone of half angle

Manuscript received January 21, 1980.

The author was with General Motors Research Laboratories, Warren, MI 48090 . He is now with the Department of Physics, University of Texas, Austin, TX 78712.

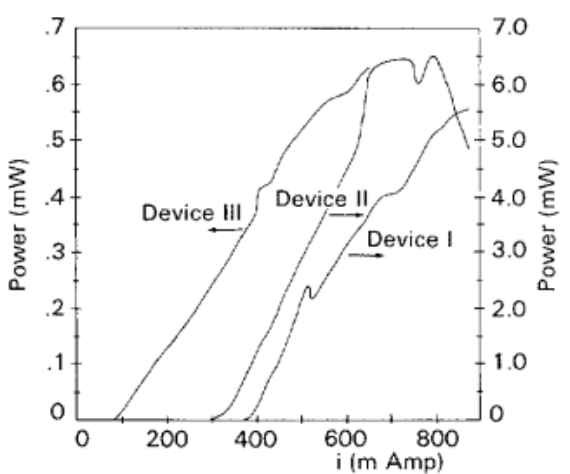

Fig. 1. Single-end output power versus injection current for $\mathrm{CW}$ operation of three lead-sulfide-selenide lasers. The scale on the right refers to devices I and II, and the left scale is for device III.

$25^{\circ}$. An image of the near-field diode emission is formed by a $\mathrm{ZnSe}$ doublet of unit $f$-number and effective focal length 30.3 $\mathrm{mm}$ positioned to provide $12 \mathrm{X}$ magnification. By translating an aperture mounted to an indium-antimonide detector in the image plane, the near-field intensity distribution can be mapped.

Special attention has been given to the design and fabrication of the $\mathrm{ZnSe}$ lens to ensure that for our experiments the limiting resolution of the lens would be set by diffraction and not by aberration. Our measurements with a narrow-bandwidth infrared source demonstrate that, for an object located within $\pm 0.1^{\circ}$ of the axis of the lens, the image characteristics are determined by diffraction from the effective aperture of the lens. Since the angular divergence at the $1 / e^{2}$ points for our diode lasers operating above threshold is approximately $\pm 15^{\circ}$, the $F / 1$ lens minimizes the effect of truncation of the laser beam at the lens aperture [3], i.e., the lens is underfilled by the laser emission.

The position of the image plane of the diode emission is determined in the fashion described by Kajimura et al. [5] . While no investigation of possible astigmatism of the laser emission was made, motion of the detection aperture parallel to the optic axis confirmed that the position of the image plane changed less than $1 \mathrm{~mm}$ with the injection level above or below threshold, indicating that the object plane coincides with the emitting face of the diode to within $10 \mu \mathrm{m}$.

Fig. 1 shows the output power from one face of the diode laser as a function of injection current for each of the three lasers. Just above threshold the output is linear in current and in this region the differential external quantum efficiencies [4] for the lasers I, II, and III are 0.14, 0.12, and 0.009, respectively. 


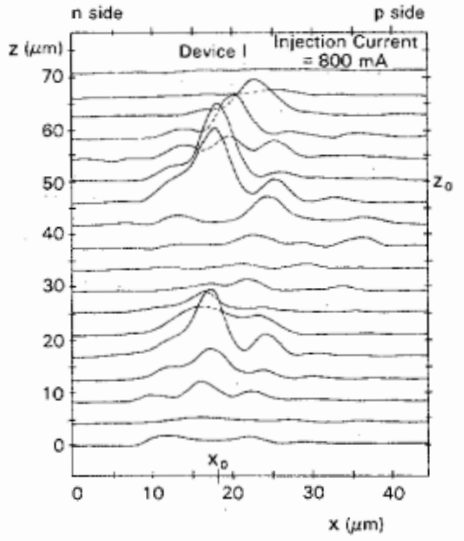

Fig. 2. Near-field intensity distribution for device I. The height of a given trace along $x$ is proportional to the near-field intensity at the coordinates $(x, z)$ perpendicular and parallel to the junction. The origin for $x$ and $z$ is arbitrary.

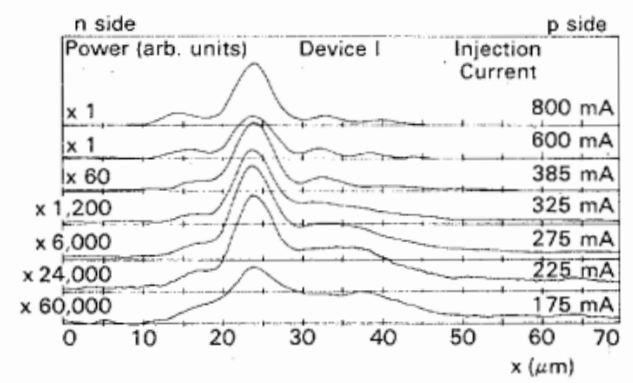

Fig. 3. Dependence of the near-field emission on injection current along a direction $x$ perpendicular to the junction. The position parallel to the junction is fixed at $z_{0}$ as indicated in Fig. 2 .

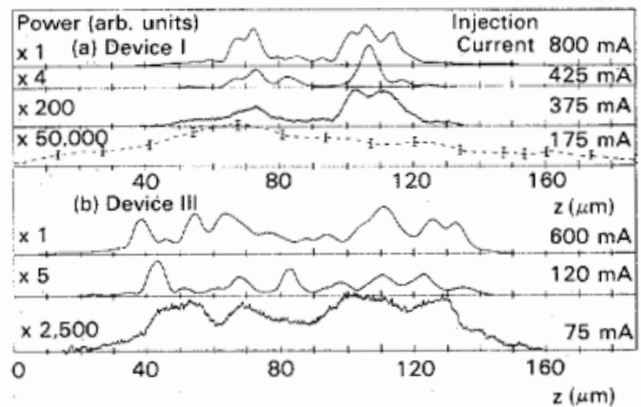

Fig. 4. Dependence of the near-field emission on injection current along a direction $z$ parallel to the junction: (a) Device I for $x_{0}$ as indicated in Fig. 2(b); device III with $x$ fixed for maximum intensity. The vertical scales in (a) and (b) are unrelated.

In Figs. 2-4 we present the results of measurements of the near-field intensity distributions. The traces were generated by translating an aperture in the image plane of the diode emission along a direction $x$ perpendicular to the p-n junction or along a direction $z$ parallel to the junction. For any one of the scans, the peak height is proportional to the laser intensity at the coordinates $(x, z)$. While the origin for $x$ and $z$ is arbitrary in each of the figures, the values given on the axes take into account the lens magnification and represent distances in the object plane.

Fig. 2 is a map of the near-field emission from device I obtained by scanning along $x$ at successive values of $z$. The oscillatory structure evident in Fig. 2 is not an artifact of the optical system but is rather characteristic of the diode emission.
Sufficiently far below threshold the emission broadens and the structure shown in Fig. 2 is lost.

The dependence of the intensity distribution perpendicular to the p-n junction on the injection level is shown in Fig. 3. These scans are made at a fixed distance $z_{0}$ along the junction, as indicated in Fig. 2. Below threshold the distribution broadens toward the p-side, presumably as a result of the diffusion of minority carriers into the p-region with some spontaneous recombination lifetime. If from Fig. 3 we estimate the diffusion length $d$ to be $20 \mu \mathrm{m}$ and take for the electron mobility $\mu$ the value $7 \times 10^{4} \mathrm{~cm}^{2} / \mathrm{V} \cdot \mathrm{s}[6]$, we can use the relation [4]

$$
d=\sqrt{\frac{k T}{e} \mu \tau}
$$

to compute a lifetime $\tau$ of $2 \times 10^{-8} \mathrm{~s}$ for a junction temperature of $20 \mathrm{~K}$. The asymmetry of the profile in Fig. 3 below threshold is due to the shallow $(10-15 \mu \mathrm{m})$ junction relative to the n-type layer.

In addition to this value for $d$, the broadening of the diode emission below threshold shown in Fig. 3 allows us to estimate the distributed loss coefficient $\alpha$ for the laser as follows. At the threshold for laser oscillation, the gain of the cavity is equal to the sum of the distributed and reflection losses [4]

$$
g_{\text {th }}=\alpha+\frac{1}{l} \ln \left(\frac{1}{R}\right)
$$

with $l$ as the cavity length and $R$ equal to the reflectivity of the cleaved facets. Slightly below threshold the gain is less than $g_{\text {th }}$ but is still larger than $\alpha$. Hence there is a net gain for a single pass along the cavity, and the dominant contribution to the laser output will come from stimulated emission along the cavity axis and not from spontaneous emission. On the other hand, for $g<\alpha$ there will be a net absorption along the cavity axis, and the output of the device will be dominated by the spontaneous recombination near the end reflector.

The transition from the domain $g>\alpha$ to $g<\alpha$ should be accompanied by a change in the near-field emission profile, from a structure that is characteristic of any passive optical guiding mechanism along the laser axis to one that arises from spontaneous recombination near the output face of the diode. If in Fig. 3 we take $i=175 \mathrm{~mA}$ as the point where the gain and distributed losses are approximately equal, we find with the help of (2) that

$$
\frac{g(175 \mathrm{~mA})}{g_{\text {th }}(385 \mathrm{~mA})}=\frac{\alpha}{\alpha+\frac{1}{l} \ln \frac{1}{R}}
$$

For low temperature operation of the diode laser, we expect that the gain and current density $J$ will be linearly related, $g=\beta J[7]$, although some evidence suggests this relationship may not be valid for our lasers [8]. Combining this relationship with (3), we find that $\alpha \approx 25 \mathrm{~cm}^{-1}$ and $\beta \approx 0.09$ $\mathrm{cm} / \mathrm{A}$ for an assumed uniform current flow and for a reflectivity $R=0.4$ [9]. These estimates for $\alpha$ and $\beta$ are in reasonable agreement with the work of Ralston et al. [1] for 
$\mathrm{Pb}_{0.88} \mathrm{Sn}_{0.12} \mathrm{Te}$ and are in line with the values quoted for GaAs lasers [4].

For operation above threshold our interpretation of the structure shown in Fig. 3 follows that by Zachos and Ripper [10]. The process used to form the p-n junction [2] in our Iasers gives rise to an approximately linear change in the difference of the number of acceptors minus the number of donors as we move over $30 \mu \mathrm{m}$ from n-type to p-type material [11]. For bulk carrier concentrations of $N_{2}=2 \times 10^{18} /$ $\mathrm{cm}^{3}$ [2], the refractive index $n_{2}$ in the bulk will be less than the index $\bar{n}$ of an undoped sample by an amount [12]

$$
n_{2}=\bar{n}\left[1-\frac{N_{2} e^{2}}{2 m^{*} \omega^{2} \epsilon_{0} \bar{n}^{2}}\right] \text {. }
$$

Likewise, the index $n_{1}$ in the compensated region of the junction will differ from $\bar{n}$ due to the carrier concentration $N_{1}$ at the junction. Of course $N_{1}$ is somewhat ill defined, but if we assume that $N_{1}=5 \times 10^{17} / \mathrm{cm}^{3}$, we find a change in index $\left(n_{2}-n_{1}\right)$ in moving from the junction to either the p- or ntype material given by

$$
\frac{n_{2}-n_{1}}{\bar{n}}=-\frac{\left(N_{2}-N_{1}\right) e^{2}}{2 m^{*} \omega^{2} \epsilon_{0} \bar{n}^{2}}=-0.011
$$

Here $m^{*}$ is the electron or hole effective mass, either of which we take to be 0.08 times the electron mass [6], $\omega$ is the laser frequency of $4 \times 10^{14} \mathrm{rad} / \mathrm{s}, \epsilon_{0}$ is the vacuum permittivity, and $\bar{n}$ is chosen to be 4.5 [9]. If we now incorporate this change in index into the model of Zachos and Ripper [10] and assume that the change occurs over a distance of $15 \mu \mathrm{m}$ on either side of the junction, we find that the structure in the near field perpendicular to the junction should be contained in a Gaussian envelope of full width at half maximum of 6.8 $\mu \mathrm{m}$. This number is in agreement with the widths of $5-8 \mu \mathrm{m}$ observed for all three lasers and is reasonably insensitive to our original choice for $N_{1}$.

Turning our attention to the structure of the near field parallel to the junction, we see from Fig. 4 that the lasers studied have quite different properties. Devices I and II have similar emission profiles, as illustrated in Fig. 4(a). These scans were taken along $z$ at the fixed position $x_{0}$ indicated in Fig. 2. Note that as the current is increased from 425 to $800 \mathrm{~mA}$, the peak centered around $z=115 \mu \mathrm{m}$ broadens appreciably, possibly due to incomplete gain saturation in the wings of the initially narrow intensity distribution [4]. The peak located at $70 \mu \mathrm{m}$ is relatively broad at all values of current above threshold and makes an increasingly larger contribution to the laser emission as the current is raised.

The data shown in Fig. 4(b) are for device III and were taken at a fixed position $x$ corresponding to the maximum intensity perpendicular to the junction. Below threshold there is uniform emission over the entire width of the junction. However, above threshold this emission does not collapse as in Fig. 4(a), but rather remains broad with many peaked regions. We note that for each of the three lasers studied, the width of the spontaneous emission region as determined from the image dimensions and lens magnification agrees with the measured geometric width of the diode along $z$.
The mechanism responsible for the guiding of the laser emission parallel to the junction is difficult to identify. While the peaked structure of devices I and II could be due to material inhomogeneity, the similarity of the near-field patterns that we have observed for these two lasers suggests that the explanation should be related instead to the spatial dependence of the laser gain and refractive index on injection current [13]. It has been suggested that variations in the real part of the refractive index are the dominant guiding mechanism in the lead-salts due to the large contribution to the dielectric constant by free carries in these materials [14]. Our observation of an apparent lack of shift in object position with injection current supports this suggestion, since contributions from the imaginary part of the index, i.e., gain guiding, would tend to produce astigmatism in the laser emission [15]. The cause of the marked difference in threshold, output power, and nearfield emission between device III and the other two lasers is not known, but we believe that it is related to the technique used to mount the laser [11].

Of course it is possible that the structures we have observed in the near-field emission result from carrier confinement rather than from optical confinement. However, our data seem to support the contention of optical guiding for the following reasons. In the first place the oscillatory structure present in the near field in the vicinity of and above threshold is certainly suggestive of the excitation of the high-order mode structure of a dielectric waveguide. Measurements that we have made of the spectral distribution of the laser emission exhibit frequency spacings consistent with this interpretation and with calculations based on the work of Zachos and Ripper [10]. Secondly, if the structure shown in Fig. 3 were due to the finite lifetime for stimulated recombination rather than to optical confinement, one would expect this structure to continue to narrow above threshold as the stimulated recombination lifetime decreases. Such narrowing above threshold is not observed within the limit of our resolution, even though we do infer a decreased radiative lifetime from our measurements of the differential junction voltage and cavity emission frequencies.

We have made measurements of the near field in the regions near the "kinks" shown in Fig. 1 in an attempt to correlate these nonlinearities with changes in the lateral or transverse mode structure of the laser [4]. Referring to the power output curve for device I in Fig. 1, we have found that the near field is unchanged at currents slightly below and above the "kink" at $520 \mathrm{~mA}$. Both emitting regions parallel to the junction for this device suffer the same fractional change in intensity at the "kink." This result is somewhat surprising since such "kinks" are normally associated with changes in the lateral mode number of the laser. However, we find no such changes in this device at the "kink," suggesting that either they are unresolved by our optics or unassociated with the power change. For device II we observe a drastic effect in the near field around $800 \mathrm{~mA}$ that coincides with the steep fall off in laser output power shown in Fig. 1. The peaked structure of the type shown in Fig. 2 is lost above $800 \mathrm{~mA}$ as the emission spreads into a broad region $25 \times 35 \mu \mathrm{m}$ perpendicular and parallel to the junction. 
Finally we note that the positions of the peaks in the emission profiles are invariant with respect to cycling of the injection current for all three lasers. The relative importance of the regions of laser activity does vary with current, but the variations are reproducible and are such that the positions of the active regions remain unchanged. Each laser has been thermally cycled from room temperature to $12 \mathrm{~K}$ at least five times with no noticeable change in the near field, although the power versus current curves do change somewhat.

In summary, we have presented data displaying the nearfield emission profiles of three lead-sulfide-selenide homojunction lasers. We have discussed the stability of the observed distributions with respect to changes in injection current and have estimated the distributed losses of the laser. Optical confinment perpendicular to the junction seems to be a result of static index guiding due to the characteristics of the homojunction, while the source of mode guiding parallel to the junction has not been identified.

\section{ACKNOWLEDGMENT}

W. Lo of General Motors Research Laboratories provided the lasers used in these experiments and discussions with him have been most helpful. The technical assistance of T. H. Van Steenkiste is gratefully acknowledged.

\section{REFERENCES}

[1] R. W. Ralston, I. Megailis, A. R. Calawa, and W. T. Lindley, "Stripe-geometry $\mathrm{Pb}_{1-x} \mathrm{Sn}_{x} \mathrm{Te}$ diode lasers," IEEE J. Quantum Electron., vol. QE-9, pp. 350-356, 1973.

[2] W. Lo and D. E. Swets, "Diffused homojunction lead-sulfide- selenide diodes with $140 \mathrm{~K}$ laser operation," Appl. Phys. Lett., vol. 33, pp. 938-940, 1978.

[3] L. D. Dickson, "Characteristics of a propagating Gaussian beam," Appl. Opt., vol. 9, pp. 1854-1861, 1970.

[4] H. Kressel and J. K. Butler, Semiconductor Lasers and Heterojunction LEDs. New York: Academic, 1977, Appendix B.

[5] T. Kajimura, T. Kuroda, S. Yamashita, M. NaKamura, and J. Umeda, "Transverse-mode stabilized $\mathrm{Ga}_{1-x} \mathrm{Al}_{x}$ As visible diode lasers," Appl. Opt., vol. 18, pp. 1812-1815, 1979.

[6] Y. I. Ravich, B. A. Efimova, and I. A. Smirnov, Semiconducting Lead Chalcogenides. New York: Plenum, 1979, Appendix C.

[7] F. Stern, Laser Handbook, F. T. Arecchi and E. O. SchulzDeBois, Eds. Amsterdam, The Netherlands: North-Holland, 1972, pp. 425-440.

[8] J. N. Walpole, A. R. Calawa, T. C. Harmon, and S. H. Groves, "Double-heterostructure PbSnTe lasers grown by molecularbeam epitaxy with cw operation up to $114 \mathrm{~K}$," Appl. Phys. Lett., vol. 28, pp. 552-554, 1976.

[9] J. N. Zemel, J. D. Jensen, and R. B. Schoolar, "Electrical and optical properties of epitaxial films of $\mathrm{PbS}, \mathrm{PbSe}, \mathrm{PbTe}$, and SnTe," Phys. Rev., vol. 140, pp. A330 A342, 1965.

[10] T. H. Zachos and J. E. Ripper, "Resonant modes of GaAs junction lasers," IEEE J. Quantum Electron., vol. QE-5, pp. 29-37, 1969.

[11] W. Lo, private communication.

[12] R. A. Smith, Semiconductors. London, England: Cambridge Univ. Press, 1964, p. 222.

[13] F. R. Nash, "Mode guidance parallel to the junction plane of double-heterostructure GaAs lasers," J. Appl. Phys., vol. 44, pp. 4696-4707, 1973.

[14] J. N. Walpole, A. R. Calawa, R. W. Ralston, T. C. Harmon, and J. P. McVittie, "Single heterojunction $\mathrm{Pb}_{1-x} \mathrm{Sn}_{x} \mathrm{Te}$ diode lasers," Appl. Phys. Lett., vol. 23, pp. 620-662, 1973.

[15] D. D. Cook and F. R. Nash, "Gain-induced guiding and astigmatic output beam of GaAs lasers," J. Appl. Phys., vol. 46, pp. 1660$1672,1975$.

H. J. Kimble, for a photograph and biography, see page 461 of the April 1980 issue of this JournaL 